Address correspondence to: Cathy Mendelsohn, Departments of Urology and Pathology, Columbia University, 650 West 168th Street, New York, New York 10032, USA. Phone: (212) 305-1591; Fax: (212) 305-6851; E-mail: clm20@columbia.edu.

1. Chevalier, R.L. 2004. Obstructive nephropathy and the developing kidney: too little or too much angiotensin? Kidney Int. 65:1517-1518.

2. Chevalier, R.L. 1998. Pathophysiology of obstructive nephropathy in the newborn. Semin. Nephrol. 18:585-593.

3. Scott, J.E., and Renwick, M. 1988. Antenatal diagnosis of congenital abnormalities in the urinary tract. Results from the Northern Region Fetal Abnormality Survey. Br. J. Urol. 62:295-300.

4. Kume, T., Deng, K., and Hogan, B.L. 2000. Murine forkhead/winged helix genes Foxc1 (Mf1) and Foxc2 (Mfh1) are required for the early organogenesis of the kidney and urinary tract. Development. 127:1387-1395.

5. Grieshammer, U., et al. 2004. SLIT2-mediated $\mathrm{ROBO} 2$ signaling restricts kidney induction to a single site. Dev. Cell. 6:709-717.

6. Basson, M.A., et al. 2005. Sprouty1 is a critical regulator of GDNF/RET-mediated kidney induction. Dev. Cell. 8:229-239.

7. Batourina, E., et al. 2005. Apoptosis induced by vitamin A signaling is crucial for connecting the ureters to the bladder. Nat. Genet. 37:1082-1089.

8. Mackie, G.G., and Stephens, F.D. 1975. Duplex kidneys: a correlation of renal dysplasia with position of the ureteral orifice. J. Urol. 114:274-280.

9. Ichikawa, I., Kuwayama, F., Pope, J.C., Stephens, F.D., and Miyazaki, Y. 2002. Paradigm shift from classic anatomic theories to contemporary cell biological views of CAKUT. Kidney Int. 61:889-898.

10. Wall, B., and Wachter, E. 1952. Congenital ureteral valve: its role as a primary obstructive lesion: classification of the literature and report of an authentic case. J. Urol. 68:684-690.

11. Wu, X.R., et al. 1994. Mammalian uroplakins. A group of highly conserved urothelial differentiation-related membrane proteins. J. Biol. Chem. 269:13716-13724.

12. Kong, X.T., et al. 2004. Roles of uroplakins in plaque formation, umbrella cell enlargement, and urinary tract diseases. J. Cell Biol. 167:1195-1204.

13. Hu, P., et al. 2001. Ablation of uroplakin III gene results in small urothelial plaques, urothelial leakage, and vesicoureteral reflux. Urology. 57:117.

14. Yu, J., Carroll, T.J., and McMahon, A.P. 2002. Sonic hedgehog regulates proliferation and differentiation of mesenchymal cells in the mouse metanephric kidney. Development. 129:5301-5312.

15. Chang, C.P., et al. 2004. Calcineurin is required in urinary tract mesenchyme for the development of the pyeloureteral peristaltic machinery. J. Clin. Invest. 113:1051-1058. doi:10.1172/JCI200420049.

16. Miyazaki, Y., Oshima, K., Fogo, A., Hogan, B.L., and
Ichikawa, I. 2000. Bone morphogenetic protein 4 regulates the budding site and elongation of the mouse ureter. J. Clin. Invest. 105:863-873.

17. Raatikainen-Ahokas, A., Hytonen, M., Tenhunen, A., Sainio, K., and Sariola, H. 2000. BMP-4 affects the differentiation of metanephric mesenchyme and reveals an early anterior-posterior axis of the embryonic kidney. Dev. Dyn. 217:146-158.

18. Sukegawa, A., et al. 2000. The concentric structure of the developing gut is regulated by Sonic hedgehog derived from endodermal epithelium. Development. 127:1971-1980.

19. Roberts, D.J., Smith, D.M., Goff, D.J., and Tabin, C.J. 1998. Epithelial-mesenchymal signaling during the regionalization of the chick gut. Development. 125:2791-2801

20. Airik, R., Bussen, M., Singh, M.K., Petry, M., and Kispert, A. 2006. Tbx18 regulates the development of the ureteral mesenchyme. J. Clin. Invest. 116:663-674. doi:10.1172/JCI26027.

21. Kim, B.M., Buchner, G., Miletich, I., Sharpe, P.T., and Shivdasani, R.A. 2005. The stomach mesenchymal transcription factor Barx1 specifies gastric epithelial identity through inhibition of transient Wnt signaling. Dev. Cell. 8:611-622.

22. Liang, F.X., et al. 2005. Cellular basis of urothelial squamous metaplasia: roles of lineage heterogeneity and cell replacement. J. Cell Biol. 171:835-844.

23. Lipschutz, J.H., Young, P., Taguchi, O., and Cunha, G.R. 1996. Urothelial transformation into functional glandular tissue in situ by instructive mesenchymal induction. Kidney Int. 49:59-66.

\title{
Ghrelin receptor mutations — too little height and too much hunger
}

\author{
Birgitte Holst and Thue W. Schwartz
}

Laboratory for Molecular Pharmacology, Panum Institute, University of Copenhagen, Copenhagen, Denmark.

\begin{abstract}
The ghrelin receptor is known from in vitro studies to signal in the absence of the hormone ghrelin at almost $50 \%$ of its maximal capacity. But, as for many other 7-transmembrane receptors, the in vivo importance of this ligand-independent signaling has remained unclear. In this issue of the JCI, Pantel et al. find that a natural mutation in the ghrelin receptor, Ala204Glu, which is associated with a selective loss of constitutive activity without affecting ghrelin affinity, potency, or efficacy, segregates in $\mathbf{2}$ families with the development of short stature (see the related article beginning on page 760). By combination of the observations from this study with those related to the phenotype of subjects carrying another natural ghrelin receptor mutation, Phe279Leu, having identical molecular-pharmacological properties, it is proposed that selective lack of ghrelin receptor constitutive signaling leads to a syndrome characterized not only by short stature, but also by obesity that apparently develops during puberty.
\end{abstract}

Seven-transmembrane segment (7TM) or $\mathrm{G}$ protein-coupled receptors can signal without any agonist present (1). This

Nonstandard abbreviations used: CB1, cannabinoid type $1 ; \mathrm{GH}$, growth hormone; 7TM, 7-transmembrane segment.

Conflict of interest: The authors have declared that no conflict of interest exists.

Citation for this article: J. Clin. Invest. 116:637-641 (2006). doi:10.1172/JCI27999. phenomenon is a simple reflection of the molecular activation mechanism where the receptor is in an equilibrium between inactive and active conformations and can relatively easily access the active conformation without the presence of an agonist (2). The degree of ligand-independent or constitutive signaling varies among receptors but in most cases only represents a small fraction of the maximal signaling capacity and is consequently generally ignored. However, among receptors involved in the control of appetite and energy expenditure, such constitutive activity appears to have important functional consequences. The cannabinoid type 1 (CB1) receptor, which is the target for the novel antiobesity drug rimonabant - acting as a combined antagonist and inverse agonist - and the ghrelin receptor both signal with around 50\% activity in the absence of ligand (3-5). Nevertheless, the physiological importance of such constitutive signaling has been very hard to establish in vivo, mainly because of the lack of appropriate pharmacological tools. Inverse agonists, i.e., ligands that inhibit the constitutive receptor signaling, in most cases also act as antagonists, i.e., they also block the action of the endogenous agonist. Thus, it is very hard in the in vivo setting to differentiate between an effect on constitutive receptor signaling and a blocking effect on receptor access to an endogenous ligand. In this issue of 


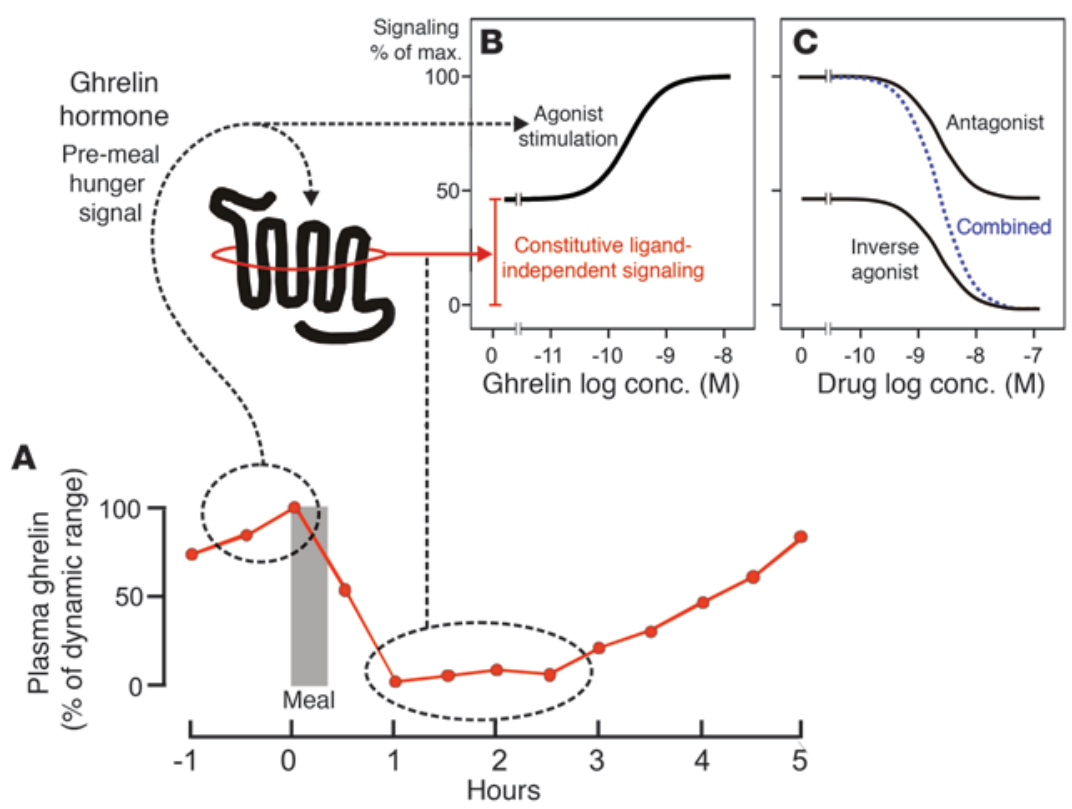

Figure 1

Constitutive and hormone-mediated signaling of the ghrelin receptor in relation to the dynamic pattern of ghrelin secretion. (A) Variations in plasma ghrelin concentration (conc.) depicted as a dynamic range in relation to a meal. Note the pre-meal surge in ghrelin secretion followed by inhibition related to the presence of food in the upper gastrointestinal tract. Illustration based on work of Cummings and coworkers $(12,13)$. (B) Illustration of the almost $50 \%$ constitutive, ligand-independent signaling of the ghrelin receptor as measured, for example, in inositol phosphate accumulation assays and the agonist response mediated by the ghrelin hormone (4). To the upper left is indicated that the high constitutive ghrelin receptor signaling is expected to be dominant in the inter-meal period, while the ghrelin-mediated signaling is most important in the fasting state and pre-meal situation (10). (C) Diagram of the theoretical effect of an inverse agonist, which inhibits constitutive signaling; the effect of a pure antagonist, which blocks the agonist-mediated signaling without affecting the constitutive signaling; and the effect of a combined antagonist and inverse agonist (dotted curve in blue), which blocks both the agonist-induced signaling and the constitutive signaling. It should be noted that for 7TM receptors in general, antagonists generally also act as inverse agonists, a phenomenon whose salience depends on the receptor displaying a reasonable degree of constitutive signaling. However, compounds have been described — for example, in the ghrelin receptor system — that are significantly more potent as inverse agonists than as antagonists (4).

the JCI, Pantel and coworkers provide very strong evidence for a physiological role of the constitutive activity of the ghrelin receptor in the development of normal height in humans (6).

\section{Agonist-dependent and constitutive signaling of the ghrelin receptor}

Ghrelin is a hormone and neuropeptide involved in growth hormone $(\mathrm{GH})$ release and control of food intake and energy expenditure (7-9). Besides being a transmitter in discrete neuronal networks in, for example, the hypothalamus, ghrelin functions as a hormonal "hunger signal" from enteroendocrine cells in the stomach to various target cells located in afferent vagal neurons, the brain stem, and the arcuate nucleus of the hypothalamus $(10,11)$. The dynamic pattern of ghre- lin concentrations in plasma is opposite that of gastrointestinal tract hormones in general: a surge before the first meal of the day is followed by a prolonged nadir caused by the inhibitory effect of food being present in the upper gastrointestinal tract (Figure 1) $(12,13)$. Thus, it has been suggested that, between meals, constitutive GH secretagogue receptor activity could play an important role in modulating the orexigenic signals in the regulatory pathways that are integrating anorexigenic signals such as leptin, insulin, and peptide $\mathrm{YY}_{3-36}$, etc. (10). The amount of constitutive signaling is directly proportional to the expression level of the receptor, and the expression of the ghrelin receptor is - like that of the CB1 receptor - highly regulated, for example by fasting $(10,14)$.

\section{Selective loss of ghrelin receptor constitutive activity associated with short stature}

In a systematic search among subjects with short stature, Pantel and coworkers found an identical missense mutation - Ala204Glu - in the ghrelin receptor in 2 unrelated Moroccan subjects, one with idiopathic short stature and another with isolated GH deficiency (6). Surprisingly, ghrelin bound with normal (high) affinity and stimulated signal transduction with normal potency and normal apparent efficacy at the Ala204Glu receptor variant. Importantly, however, the characteristic high constitutive signaling activity of the receptor was eliminated by the mutation, and the receptor numbers at the cell surface were somewhat lower compared with the wild-type (6). In the 2 families, the mutation lacking constitutive activity segregated with short stature in a dominant manner with a penetrance of approximately $70 \%$ (6). Interestingly, one of the probands, who developed short stature during the first years of her life and subsequently developed obesity around puberty, was homozygotic for the mutation because of a consanguineous relationship between her heterozygotic parents, both of whom were not only very short, but also obese. Nevertheless, Pantel and coworkers were - based on the limited patient material - not able to associate the obese phenotype directly to the receptor mutation (6).

\section{The loss of ghrelin receptor constitutive activity may be part of a broader syndrome}

The Ala204Glu allele of the ghrelin receptor had already been described previously in a German subject by Hinney, Hebebrand, and coworkers, but without any functional characterization (15). In a study on the occurrence of ghrelin receptor variants in populations of obese and underweight subjects, as well as subjects with short stature, they identified 2 novel missense variants of the ghrelin receptor of which the Ala204Glu variant was found to be heterozygous in a very obese child. This observation adds further support to the notion that obesity could be part of the phenotype of carriers of the Ala204Glu variant of the ghrelin receptor.

The other novel, uncharacterized ghrelin receptor variant reported in the German study - a Phe279Leu mutation - was identified in a child with short stature 


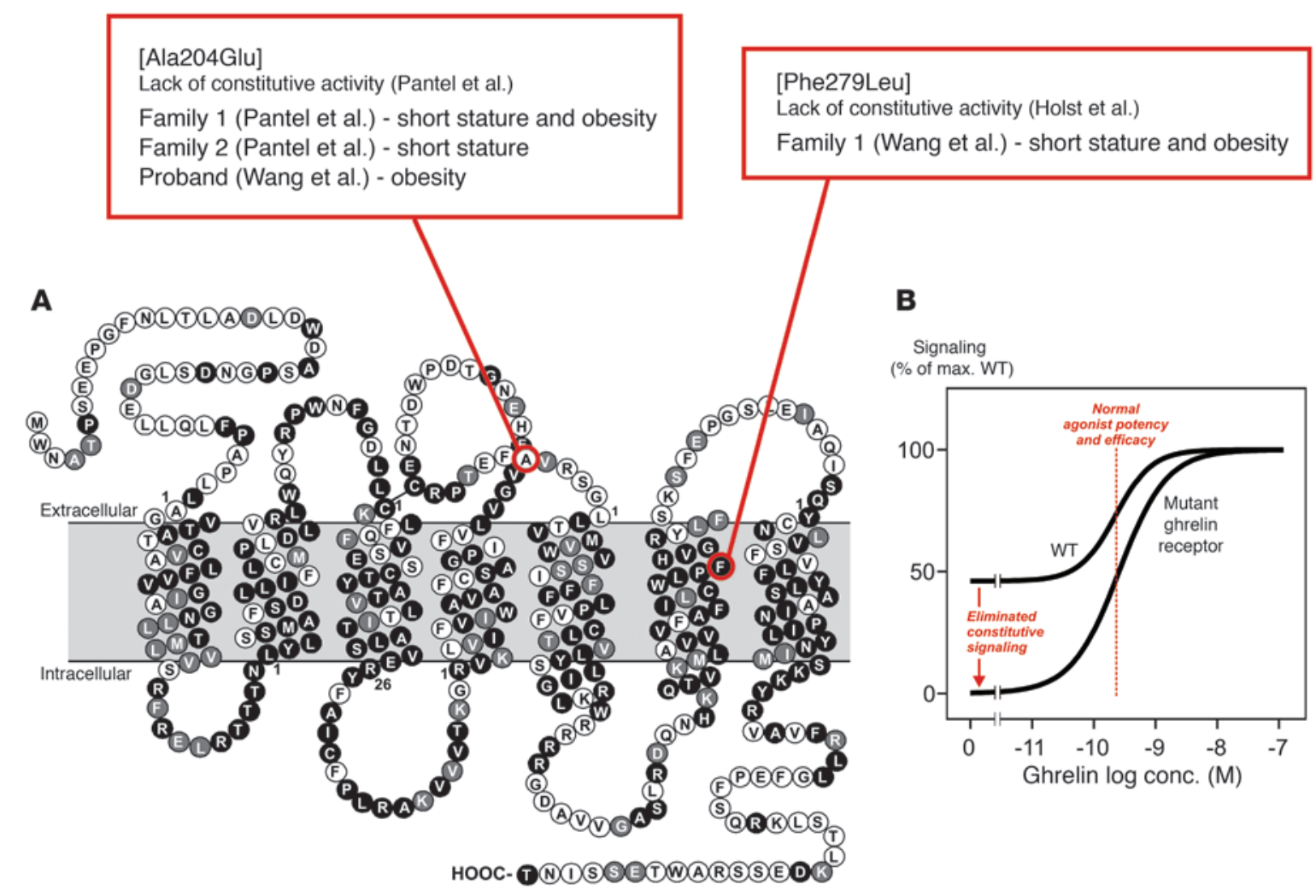

Figure 2

Natural mutations selectively eliminating the constitutive signaling of the ghrelin receptor that are associated with short stature and possible obesity. (A) Serpentine model of the human ghrelin receptor in which residues that are identical (white on black) or structurally conserved (white on gray) between the ghrelin receptor and its closest homologue, the motilin receptor, are indicated. Note that a Cys residue in the middle of extracellular loop 2 forms a disulfide bridge with a Cys residue at the extracellular end of transmembrane segment III (TM-III), which is a highly conserved structural feature of 7TM receptors. The location of the Ala204Glu mutation in the part of extracellular loop 2 connecting the extracellular end of TM-III with TM-V, which Pantel et al. describe in this issue of the $J C l(6)$, is highlighted with a red circle (6). As indicated in the red box at the top left, this mutation selectively eliminates constitutive signaling by the ghrelin receptor and is associated with short stature (6). This mutation has previously been identified in a very obese child by Wang et al. (15). Also highlighted with a red circle is the location of the Phe279Leu mutation in TM-VI. The constitutive activity of the ghrelin receptor is highly dependent on the presence of an aromatic residue at position VI:16 (16), and Wang et al. found the Phe279Leu mutation in a child with short stature and in his mother, who was also of short stature $(157 \mathrm{~cm})$ and who was very obese $(\mathrm{BMI}=34.6)(15)$. (B) Schematic diagram of the effects of these natural mutations in eliminating the constitutive signaling of the ghrelin receptor (red arrow to the left) without affecting the potency or efficacy of the natural ligand, ghrelin (dotted vertical line corresponding to the $\mathrm{EC}_{50}$ ).

of severity comparable to that seen in the French-Moroccan study (15). However, during a systematic structure-function analysis of residues located in the main ligand-binding pocket of the ghrelin receptor, Phe279 (PheVI:16) was recently identified as being especially important for the constitutive signaling activity of the receptor (16). That is, Phe279 is part of an unusual cluster of aromatic residues located on the inner faces of transmembrane segments VI and VII, which are essential in holding the ghrelin receptor in the active conformation. Removal of the aromaticity at position 279 (VI:16) selectively eliminates the constitutive activity of the receptor (16). Thus, the German child with short stature carries a differ- ent ghrelin receptor mutant - Phe279Leu - which, however, has a molecular-pharmacological profile similar to that of the Ala204Glu mutant found in the FrenchMoroccan subjects with a similar degree of short stature (Figure 2).

Regarding the question of whether obesity is part of the phenotype associated with a ghrelin receptor lacking constitutive activity, it is particularly interesting that the mother of the German proband, who also was a carrier of the Phe279Leu mutant receptor, was not only very short $(157 \mathrm{~cm})$ but also very obese $(\mathrm{BMI}=34.6)$ (ref. 15, and personal communication with A. Hinney and J. Hebebrand, University of Duisburg-Essen, Essen, Germany). As discussed above, obesity was frequently observed among members of the Moroccan families, especially in adult carriers (6). Interestingly, the Ala204Glu homozygotic proband was somewhat overweight as a child but became severely obese only around puberty (see Figure 3 in ref. 6). Thus, based on the 2 different but functionally similar mutations, and the 4 families in which these are found $(6,15)$, we suggest that selective loss of ghrelin receptor constitutive activity causes a syndrome of short stature and obesity, of which the obesity appears to develop around puberty.

\section{How can loss of ghrelin receptor function lead to obesity?}

It can rather easily be rationalized that loss-of-function mutations in the ghrelin 
receptor would lead to the development of short stature through reduced activity at the hypothalamic-pituitary-GH axis - although, in the present case, the precise mechanism of this is not entirely clear (6). In contrast, with respect to the control of appetite and energy expenditure, it would be expected that loss-of-function mutations in the ghrelin receptor would lead to a lean and not an obese phenotype, because ghrelin is a potent hunger signal. In accordance with this inference, recent studies in mice have shown that knockout of either the ghrelin hormone or the ghrelin receptor protects against obesity induced by a high-fat diet $(11,17,18)$.

Frequently, knockout of molecular elements in complex, redundant physiological systems will lead to compensatory changes in related pathways as a compensation for the lost function. Normally, such compensation will result in a diminished or total lack of the expected phenotype, but not an "overshoot," i.e., the development of the opposite phenotype. In the case of subjects expressing a ghrelin receptor that lacks constitutive signaling, there is, however, a special possibility regarding such compensation: the expression of the ghrelin receptor itself could become upregulated if it is normally controlled by an intracellular feedback mechanism requiring constitutive signaling. Because the mutant ghrelin receptor retains normal affinity and response to ghrelin, such an upregulation of receptor molecules could result in enhanced net ghrelin response, and, for example, a corresponding increase in hunger. These are obviously speculations that need to be tested experimentally. Nevertheless, it is interesting that loss of ghrelin receptor constitutive activity leads to the expected phenotype with respect to growth - i.e., short stature - but apparently leads to the opposite of the expected phenotype with respect to appetite and energy expenditure - i.e., obesity.

We have previously suggested that an inverse agonist, which eliminates the constitutive activity of the ghrelin receptor, would be particularly interesting as an antiobesity agent (10). However, apparent predisposition to obesity among subjects expressing a ghrelin receptor lacking constitutive activity could indicate that an inverse agonist approach in itself may not be optimal. But it is difficult to draw this conclusion, because the obesity could be a consequence of the persistent lack of constitutive activity during a particular period in the development of the carrier. Moreover, it should be noted that, in general, most inverse agonists also act as antagonists, i.e., they block the hormonemediated response. Thus, a combined antagonist/inverse agonist would perhaps be the preferred antiobesity agent in the ghrelin system.

\section{Cross-fertilization between genetic studies and systematic structure-function analysis}

The significance of Phe279 in ghrelin receptor function was initially identified through a systematic biochemical analysis (Figure 2), which could be performed relatively easily based on knowledge about the general structure and function of 7TM receptors (16). Thus, in this case, the basic, molecular-pharmacological analysis could help to elucidate the clinical phenotype of the patient. Conversely, the Ala204Glu mutation, which was found through a clinical genetic approach by screening of patients with a particular phenotype, has provided significant structure-function information about residues in extracellular loop 2 of the receptor (Figure 2). Importantly, this particular epitope would have been very difficult to identify through classical structure-functional analysis, because it is located outside the region that has been most intensively investigated in 7TM receptors. It is likely that a broad analysis of subjects with short stature - and especially those who develop obesity around puberty - will identify more mutations in the ghrelin receptor that, like Ala204Glu and Phe279Leu, selectively eliminate its constitutive signaling. In fact, a number of such mutations with the expected molecular-pharmacological phenotype have been identified through site-directed mutagenesis of the ghrelin receptor. It will be interesting to see whether some of these substitutions are in fact found as natural mutations among short, obese subjects.

\section{Constitutive 7TM receptor signaling is physiologically important}

Recently, Vaisse and coworkers showed that substitutions at the hottest spot for natural missense mutations in the melanocortin-4 receptor - Arg18 - as well as a couple of other sites in the N-terminal, extracellular segment of the receptor, surprisingly have no effect on $\alpha$-melanocyte-stimulating hormone $(\alpha-\mathrm{MSH})$ binding and no effect on
$\alpha-\mathrm{MSH}$ potency and efficacy but, instead, selectively eliminate the constitutive activity of the receptor (19). The obese phenotype of carriers of such mutations indicates that the constitutive activity of the melanocortin-4 receptor is important in vivo, conceivably in providing a tonic hypothalamic satiety signal. Similarly, the study by Pantel et al. in this issue of the JCI (6) demonstrates that the constitutive activity of the ghrelin receptor also is important in vivo, in this case in providing a tonic signal required for the development of normal height, conceivably through an effect on the GH axis (6).

These are both landmark studies, as they for the first time clearly demonstrate that constitutive signaling of 7TM receptors is not just an in vitro artifact or epiphenomenon but rather serves important functions of these receptors in vivo. Consequently, inverse agonists should be taken seriously, not only as pharmacological tools, but as important drug candidates.

\section{Acknowledgments}

The laboratories of the authors are supported by grants from The Novo Nordisk Foundation, the Danish Medical Research Council, and the European Community's Sixth Framework Program (grant LSHBCT-2003-503337). Birgitte Holst is supported by a personal stipend from the Weimann Foundation.

Address correspondence to: Thue W. Schwartz, Laboratory for Molecular Pharmacology, The Panum Institute, Blegdamsvej 3, University of Copenhagen, Copenhagen, Denmark. Phone: 45-3532-7601; Fax:45-3532-7610; E-mail: schwartz@ molpharm.dk.

1. Costa, T., and Cotecchia, S. 2005. Historical review: negative efficacy and the constitutive activity of Gprotein-coupled receptors. Trends Pharmacol. Sci. 26:618-624.

2. Schwartz, T.W., Frimurer, T.M., Holst, B., Rosenkilde, M.M., and Elling, C.E. 2006. Molecular mechanism of 7TM receptor activation: a global toggle switch model. Annu. Rev. Pharmacol. Toxicol. 46:481-519.

3. Adan, R.A., and Kas, M.J. 2003. Inverse agonism gains weight. Trends Pharmacol. Sci. 24:315-321.

4. Holst, B., Cygankiewicz, A., Halkjar, J.T., Ankersen, M., and Schwartz, T.W. 2003. High constitutive signaling of the ghrelin receptor-identification of a potent inverse agonist. Mol. Endocrinol. 17:2201-2210.

5. Leterrier, C., Bonnard, D., Carrel, D., Rossier, J., and Lenkei, Z. 2004. Constitutive endocytic cycle of the CB1 cannabinoid receptor. J. Biol. Chem. 279:36013-36021.

6. Pantel, J., et al. 2006. Loss of constitutive activity of the growth hormone secretagogue receptor in familial short stature. J. Clin. Invest. 116:760-768. doi:10.1172/JCI25303. 
7. Korbonits, M., Goldstone, A.P., Gueorguiev, M., and Grossman, A.B. 2004. Ghrelin: a hormone with multiple functions. Front. Neuroendocrinol. 25:27-68.

8. van der Lely, A.J., Tschop, M., Heiman, M.L., and Ghigo, E. 2004. Biological, physiological, pathophysiological, and pharmacological aspects of ghrelin. Endocr. Rev. 25:426-457.

9. Kojima, M., Hosoda, H., and Kangawa, K. 2004 Clinical endocrinology and metabolism. Ghrelin, a novel growth-hormone-releasing and appetitestimulating peptide from stomach. Best. Pract. Res. Clin. Endocrinol. Metab. 18:517-530.

10. Holst, B., and Schwartz, T.W. 2004. Constitutive ghrelin receptor activity as a signaling set-point in appetite regulation. Trends Pharmacol. Sci. 25:113-117.

11. Grove, K.L., and Cowley, M.A. 2005. Is ghrelin a signal for the development of metabolic systems? J. Clin. Invest. 115:3393-3397. doi:10.1172/ JCI27211.

12. Cummings, D.E., et al. 2001. A preprandial rise in plasma ghrelin levels suggests a role in meal initiation in humans. Diabetes. 50:1714-1719.

13. Cummings, D.E., Frayo, R.S., Marmonier, C., Aubert, R., and Chapelot, D. 2004. Plasma ghrelin levels and hunger scores in humans initiating meals voluntarily without time- and food-related cues. Am. J. Physiol. Endocrinol. Metab. 287:E297-E304.

14. Burdyga, G., et al. 2004. Expression of cannabinoid CB1 receptors by vagal afferent neurons is inhibited by cholecystokinin. J. Neurosci. 24:2708-2715

15. Wang, H.J., et al. 2004. Ghrelin receptor gene: identification of several sequence variants in extremely obese children and adolescents, healthy normal- weight and underweight students, and children with short normal stature. J. Clin. Endocrinol. Metab. 89:157-162.

16. Holst, B., et al. 2004. Common structural basis for constitutive activity of the ghrelin receptor family. J. Biol. Chem. 279:53806-53817.

17. Wortley, K.E., et al. 2005. Absence of ghrelin protects against early-onset obesity. J. Clin. Invest. 115:3573-3578. doi:10.1172/JCI26003.

18. Zigman, J.M., et al. 2005. Mice lacking ghrelin receptors resist the development of diet-induced obesity. J. Clin. Invest. 115:3564-3572. doi:10.1172/ JCI26002.

19. Srinivasan, S., et al. 2004. Constitutive activity of the melanocortin- 4 receptor is maintained by its $\mathrm{N}$-terminal domain and plays a role in energy homeostasis in humans. J. Clin. Invest. 114:1158-1164. doi:10.1172/JCI200421927. 